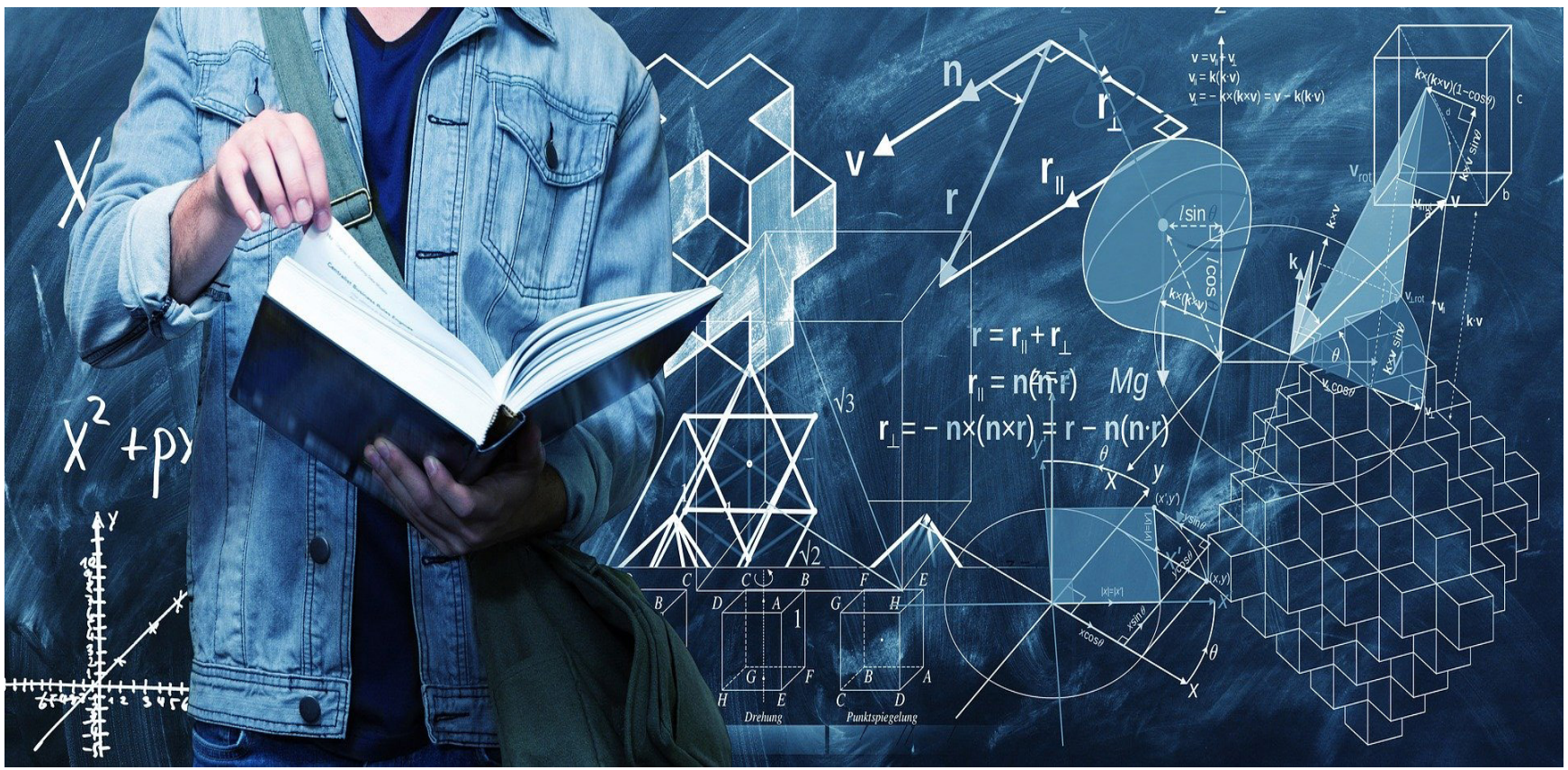

\title{
Avance de los factores protectores de la salud a través de la actividad instructiva en la enseñanza de la matemáticas
}

\section{Advancement of the protective factors of health through instructional activity in the teaching of mathematics}

Derling Isaac Mendoza-Flores

Licenciado en educación, dimf152008@gmail.com, Investigador educativo de Instituto Educativo Santo Domingo de Guzmán. Ecuador. Orcid: https://orcid.org/0000-0003-0805-4751, Quito - Ecuador.

Luz Marina Flores-Rodríguez

Especialista en Neumonología. luzmarin_21@hotmail.com,

Docente investigadora de la Universidad San Francisco de Quito. Orcid: https://orcid.org/0000-0002-7637-3079, Quito - Ecuador

\section{José Derling Velazco-Mendoza}

Doctor en Educación, derling969@gmail.com,

Docente Investigador de la Universidad Nacional de Educación UNAE. Orcid: http://orcid.org/0000-0001-8275-3687, Chuquipata - Ecuador. 


\section{Resumen}

El articulo investigativo, tuvo como objetivo proponer actividades dirigidas para la promoción de los factores protectores de la salud a través de la actividad formativa en la enseñanza de la matemática dirigida a los estudiantes del Colegio Giordano Bruno de la ciudad de Quito Ecuador (CGB), la misma se enfocó dentro de un diseño de campo de carácter descriptivo, bajo la modalidad de proyecto factible, la población se delimito por veinte (20) estudiantes que estudian en la institución antes descrita, para recopilar la información se aplicó la técnica de la observación y un cuestionario después de recopilado los datos de acuerdo a los hallazgos se elaboraron las conclusiones del diagnóstico, las cuales fomentaron la necesidad de la conformación de una propuesta para dar respuesta a los objetivos planteados. Dentro de la misma, se enmarcaron una serie de actividades que buscan transformar la práctica pedagógica ejecutada por los docentes asumiendo diferentes espacios de aprendizajes que realcen el proceso de aprendizaje que se requiere en la educación matemática afianzado a la salud. Asimismo, propiciar procesos participativos e innovadores que faciliten el conocimiento pero que a su vez sea pertinente con las necesidades e intereses de los estudiantes como los de su contexto. Por consiguiente, dar respuestas a cualquier necesidad relacionada con la salud debe ser el compromiso de la universidad como ente formador en la proyección de ambientes que propicie el sentido de pertenencia efectiva entre la institución, estudiantes y comunidad generando un clima de alta productividad.

\section{Palabras} protectores, matemática, clave:

$\begin{array}{cc}\text { clave: } & \text { Factores } \\ \begin{array}{c}\text { Salud, Enseñanza } \\ \text { investigación, la la }\end{array} & \text { Ecuador. }\end{array}$

\section{Abstract}

The objective of the research article was to propose activities aimed at promoting health protection factors through training activities in the teaching of mathematics to students at Giordano Bruno School in the city of Quito, Ecuador (CGB), the population was delimited by twenty (20) students that study in the institution before described, to gather the information the technique of the observation was applied and a questionnaire after gathered the data according to the findings the conclusions of the diagnosis were elaborated, which promoted the necessity of the conformation of a proposal to give answer to the proposed objectives. Within this, a series of activities were framed that seek to transform the pedagogical practice carried out by teachers by assuming different learning spaces that enhance the learning process required in healthenhanced mathematics education. Likewise, to foster participatory and innovative processes that facilitate knowledge but at the same time are relevant to the needs and interests of students as well as those of their context. Therefore, providing answers to any need related to health should be the commitment of the university as a training entity in the projection of environments that promote a sense of effective belonging between the institution, students and community, generating a climate of high productivity.

Keywords: Protective factors, Health, Teaching mathematics, research, Ecuador. 


\section{0}

Introducción

La [1] sugiere la utilización de diferentes elementos que puedan utilizarse para descubrir aprendizajes, partiendo del concepto de la integralidad de los contextos y espacios que se proyectan en las instituciones educativas. Sustentándose según el Art. 9 de [1] y el buen vivir. "La educación superior es condición indispensable para la construcción del derecho del buen vivir, en el marco de la interculturalidad, del respeto a la diversidad y la convivencia armónica con la naturaleza". Igualmente, el Art. 13. Con base a las funciones del sistema de educación superior. Son funciones del Sistema de [1] "Promover el respeto de los derechos de la naturaleza, la preservación de un ambiente sano y una educación y cultura ecológica”.

En este sentido, la formación en la competencia matemática, cobra especial relevancia, considerando que la preparación en el análisis, el álgebra, la geometría y porque no, la física; cómo éstas se definen y se integran permite una especialización superior, sea para enfrentar una carrera universitaria técnico científica, sea para enfrentar un mundo social basado en la innovación tecnológica que conduzcan al estudiante a la resolución de problemas cotidianos y situaciones reales [2-3], para ello es necesaria la orientación del docente, en la medida que logre a través de una evaluación contextualizada podrá transformar el proceso formativo para que sea significativo, de mayor alcance para el estudiante y le que permitan el desarrollo integral y científico como la funcionalidad de los procesos del entorno basados en una estructura propia que requiere para fortalecer los niveles de exigencias de la sociedad en cuanto a la enseñanza de las matemáticas y la salud [4].

Por consiguiente, enfatizar la promoción de factores protectores para la salud a través de experiencias y vivencias con otros, permite la integración de los estudiantes y el entorno en las ciencias numéricas, para que el CGB fortalezca en los estudiantes valores necesarios para mantener las condiciones de vida y superación intelectual.

La necesidad de construir propuestas dentro de la institución para la enseñanza de la matemática coherente con la realidad determina el abordaje de la salud en el contexto académico. A tal efecto, promover los factores protectores de la salud va más allá de la ausencia de enfermedades debe implicar reflexiones del porque y para que de las acciones que se promueven y que en este caso están en el espacio social de la salud entendiéndose este como la vida. Por consiguiente, las actividades que se ejecuten deben estar orientadas hacia procesos preventivos considerados como uno de los elementos efectivos para proteger a la población. Además, entender que en una sociedad que demanda una formación que supone concepciones individuales y colectivas para adoptar actitudes preventivas más que asistenciales para actuar comunitariamente, es una necesidad donde el CGB en su proceso formativo debe ubicar objetivos que enmarquen actividades destinadas a incrementar la aptitud de los estudiantes para tomar decisiones que se requiere para resolver problemáticas de bienestar personal, social y comunitario.

Este principio debe estar inspirado en que no se puede continuar impartiendo una educación matemática como una mera información aislada del mundo cotidiano, incluyendo sólo temas de carácter abstracto y complejo, lo que contribuye a que las actuaciones de los estudiantes se den en comportamientos pasivos y mecánicos, al igual en cuanto la salud donde se recibe información y ponen muy poco para alcanzar una conciencia individual sobre la responsabilidad propia en relación con su estado de salud y un cambio 
de comportamiento y actitudes hacia otras más saludables.

Dentro de este marco de referencia, se busca que el estudiante deje de ser pasivo y tome parte activa en el mantenimiento e incremento de su propia salud. Por tanto, la institución universitaria debe aportar ideas y sobre todo una formación de comportamientos más saludables, donde integrándose los trabajos conjuntos en materia de matemática y salud se consideren que, si se ejercen procesos y controles sobre su propia salud, logrando alcanzar un estado de completo bienestar físico, mental y social.

En este orden de ideas, se desarrolla la investigación en la necesidad de poder discernir y ubicar actividades que transformen la enseñanza de la matemática y la salud repotenciando los factores protectores para generar estilos de vida sanos y aspirar a la consecución de bienestar. La misma tuvo como objetivo proponer actividades dirigidas para la promoción de los factores protectores de la salud a través de la actividad formativa de la enseñanza de la matemáticas en el CGB.

\section{Materiales y métodos}

Se utilizó el diseño de campo que, según [5] consiste en la recolección de datos directamente de los sujetos investigados o de la realidad donde ocurren los hechos, mediante el trabajo concreto del investigador. Es decir, su innegable valor reside que a través de ellos el investigador puede cerciorarse de las verdaderas condiciones que se han conseguido sus datos, haciendo posible su revisión o modificación en el caso de que surjan dudas respecto a su calidad.

De igual manera, se utilizó la investigación descriptiva que según [6] y [7], señalan tiene el propósito exponer el evento estudiado, haciendo una enumeración detallada de sus características, de modo tal que en los resultados se pueden obtener dos niveles de análisis, dependiendo del fenómeno y del propósito del investigador: un nivel más elemental, en el cual se logra una clasificación de la información de función de características comunes, y un nivel más sofisticado en el cual se ponen en relación los elementos observados a fin de obtener una descripción más detallada.

El trabajo se ubicó el proyecto factible que busca la elaboración de una propuesta encaminada a proponer actividades dirigidas para la promoción de los factores protectores de la salud a través de la actividad formativa de la enseñanza de la matemáticas. por tanto, para efectos del estudio se desarrollarán tres fases [8]:

Fase 1. Diagnóstico de la necesidad. Se indagó sobre la problemática planteada para jerarquizar las necesidades y potencialidades detectadas para su atención. De allí, que se hace necesario aplicar instrumentos para obtener información detallada de la situación que desea mejorar, es decir describir lo más objetivamente posible la realidad de acuerdo a los datos recopilado.

Fase 2. Factibilidad. Después de recopilada la información del diagnóstico, ubicado el modelo metodológico a seguir y el escenario o contexto del estudio se establecieron los criterios que permiten asegurar el alcance de los recursos que se necesitan para el desarrollo de la propuesta. La factibilidad tiene como finalidad permitir la selección entre las variantes, determinar las características técnicas de la operación, fijar los medios a implementar, establecer los consto de operación y evaluar los recursos disponibles reales y potenciales.

Fase 3. Diseño de la propuesta. En esta etapa, se elaboró el esquema general de la propuesta de acuerdo a los resultados del diagnóstico para 


\section{2}

dar solución a las necesidades encontradas. Según [9-14], la importancia radica en combinar los recursos con el desarrollo de actividades para la obtención de ciertos logros que den respuestas a los objetivos planteados para resolver la problemática encontrada en el contexto donde se delimitó la investigación. Por tanto, se elaboró un plan contentivo de actividades que son gestionadas a través de los procesos de participación, buscando el éxito de la misma.

\section{Población y Muestra}

Se consideró como población (20) estudiantes que estudian en el CGB de la ciudad de Quito, Provincia Pichincha de la República del Ecuador, por la importancia del estudio para la institución no se realizó ningún muestreo, asumiéndose el total de la población.

Técnica e instrumentos de recolección de datos

Para la recolección de información se aplicaron dos instrumentos:

La guía de observación. Al aplicarse la guía de observación, se logró registrar lo que ocurrió en una situación real, clasificando y consignando los acontecimientos pertinentes de acuerdo con los esquemas previstos y según el problema de estudio.

El cuestionario. Consta de varias alternativas conformado por tres (3) preguntas aplicado a los estudiantes.

\section{Validez y Confiabilidad del Instrumento}

El criterio de validez utilizado fue el de contenido, que según el autor [6] se refiere el grado en que un instrumento refleja un dominio específico de contenido de lo que se mide; es decir, determinando la congruencia que contienen los ítems del instrumento con los objetivos, establece la relación con las variables que se pretenden medir. Para ello, se le entregaron tres ejemplares a docentes que tienen dominio sobre la temática y el área de metodología para su revisión y sugerencias antes de su aplicación.

\section{Confiabilidad}

El criterio de confiabilidad del instrumento, se determinó en la presente investigación por el coeficiente de Alfa de Cronbach, desarrollado por Cronbach citado por [15] quien expresa que para la medición del instrumento requiere de una sola administración y es aplicable a escalas de varios valores posibles por lo que puede ser utilizado para determinar la confiabilidad en escala cuyos ítems tienen como respuestas más de dos alternativas. Para ello, se utilizó la siguiente formula:

$$
\alpha=\frac{K}{K-1} \cdot\left[1-\frac{\sum S i^{2}}{S}\right]
$$

Donde:

$a=$ Coeficiente de confiabilidad

$\mathrm{K}=$ Números de Ítems.

$\mathrm{Si}^{2}=$ Varianza de los Puntajes Totales.

$\mathrm{S}=$ Varianza.

Los resultados fueron confiables y se ubicaron en los siguientes coeficientes 0,810 . En conclusión, el coeficiente 0.810 es determinado como un valor estadístico que se encuentran en el rango 0,800-0,899 de alta confiabilidad, según los autores [15]. Por lo tanto, el instrumento aplicado presenta un elevado índice de confiabilidad para ejecutarse durante el desarrollo de la investigación. 
Resultados y discusión

En referencia, al primer ítem titulado "Se aplican estrategias numéricas participativas para la promoción de factores protectores de salud en el proceso formativo impartido en el CGB"; revisado los instrumentos las respuestas mayores se enmarcaron en la alternativa Siempre (S) con un $50 \%$ acompañada de un $25 \%$ en Casi Siempre (CS) y el $25 \%$ restante en Casi Nunca (CN). Si se asume el $75 \%$ en las alternativas altas se ratifica que los procesos de participación son adecuados para la enseñanza de la educación matemática.

En cuanto, al segundo ítem el mismo se encuentra enfocado en la información obtenida sobre la promoción del "existe un clima comunicacional para facilitar la enseñanza de la educación para la salud y las ciencias matemáticas?"; las respuestas se enmarcaron en las alternativas Siempre (S) $75 \%$ y Casi Siempre el $25 \%$, lo que da fundamento a que el clima comunicacional impartido por los docentes facilita la enseñanza de la matemática, asumiendo esta connotación de acuerdo a lo indicado por los estudiantes.

¿Por otra parte, en el tercer ítem que expresa "se percibe la indagación del desarrollo de actividades por los docentes para fomentar la integración de la comunidad educativa en los procesos de intervención en el área de salud?"; según la ubicación de las respuestas dadas las cuales hacen referencia en la categoría Siempre (S) 10\%, Casi Siempre (CS) $40 \%$ y A veces (AV) $50 \%$.

En relación a las opiniones es pertinente acotar, que aun, cuando la mitad de los porcentajes se agruparon en la alternativa $A$ veces $(A V)$ si se toma como una media central puede valorarse como respuestas positivas. Sin embargo, en el proceso de observación de la práctica pedagógica del docente en referencia a los indicadores descritos se evidencio que no utilizan metodologías inclinadas a favorecer el área de las matemáticas en relación a la salud.

Asimismo, en su planificación solamente se observó algunas temáticas en el área de salud en los docentes de la asignatura de matemáticas, pero de manera informativa y sobre temas ubicados por los docentes sin la participación de los estudiantes. De igual manera, su labor docente no está siendo acompañada por el proceso de integración y comunicación eficiente, cuando la visión solamente está encaminada en orientar al alumno dentro de un enfoque pasivo que reciba una información, pero sin abrir el espacio para la reflexión, comprensión y valoración de la necesidad de la salud.

Por otra parte, los procesos de aprendizajes y enseñanza se ejecutan considerando solamente el aula de clase, lo que infiere como lo expresa [14] que no se da el diálogo de saberes para el descubrimiento y la producción de aprendizajes que contribuya a mejorar la calidad de vida del estudiante y por ende de su entorno. Por tanto, es vital para el fortalecimiento de aprendizajes constructivos buscando el interés por una mayor salud la vinculación con otros entes del contexto escolar que pueda situar acondicionamientos reales de la vida diaria del estudiante, la dinámica cotidiana del entorno y los beneficios que representan el sector salud [16].

De acuerdo a lo recopilado de la información emitida por los estudiantes y enfocando las observaciones de la práctica pedagógica desarrollada por estos y el compartir con algunos docentes se infiere que existen contradicciones en las respuestas, cuando no se da un enfoque integral que compagine la acción de la educación con los objetivos y componentes que son elementales desarrollar para la promoción de factores protectores de la salud en la enseñanza de la matemática. Asimismo, se evidencio que se dan algunos talleres más informativos que preventivos, 


\section{4}

donde el conocimiento es reforzado de forma mecánica, sin desarrollar responsabilidades, habilidades y destrezas que contribuya adoptar estilos de vida sano [16].

Por consiguiente, no se está proyectando procesos tan esenciales como los expresados por [17] y [18], quien ratifica la necesidad de programa secuencial y planificado, que permita la experiencia estudiantil, la cual será más efectivo si existen objetivos en común.

La indagación obtenida, y comparada con el proceso de observación dio respuestas a las interrogantes donde se considera necesario fortalecer situaciones vivenciales, prácticas que induzcan a desarrollar en forma satisfactoria y significativa los aspectos elementales que abarca la educación para la salud con énfasis en diferentes factores que se encuentran involucrados en el desarrollo de la formación y enseñanza de la matemática. De allí, el aporte generado por [19] y [20] donde plantea asumir en el área de salud prácticas dirigidas no sólo a brindar conocimientos, sino a obtener información, abordar prácticas motivacionales, vivencias, espacios que se deben considerar en los diferentes factores protectores de salud para la construcción del interés por mejores estilos de la vida.

Propuesta para la promoción de los factores protectores de la salud a través de la actividad formativa de la enseñanza de la matemáticas en el CGB

\section{Objetivo General}

- Proponer actividades didácticas dirigidas para la promoción de los factores protectores de la salud a través de la actividad formativa de la enseñanza de las matemáticas.

\section{Objetivos Específicos}

- Incentivar a los docentes para la promoción de factores protectores de la salud a través de las actividades formativas científicas del CGB.

- Reorientar los procesos desarrollados para la enseñanza de la matemática hacia el desarrollo de una práctica facilitadora de mejores estilos de vida.

- Enfocar el trabajo en conjunto para la promoción de factores protectores de la salud a través de las actividades formativas de la institución.

- Brindar acompañamiento al docente para la utilización de diferentes espacios formativos dirigidos a la promoción de factores protectores de la institución objeto del estudio.

Partiendo de la necesidad de transformar la práctica pedagógica de la institución objeto del estudio en la enseñanza de la matemática, en donde el estudiante sea el eje central y pueda ser participe en la construcción de su aprendizaje, se planificaron algunas actividades que, bajo la utilización de diferentes espacios, la universidad se interrelacione con la comunidad para comprender mejor el área de salud en su contexto social que debe abordar relaciones mutuas. Demostrándose que, de forma didáctica y dinámica, la enseñanza de las ciencias numéricas, provee de una imagen más llamativa y menos aburrida para los estudiantes, quienes han de tener fobia a las matemáticas (tabla 1). 
Tabla 1. Propuesta para la promoción de los factores protectores de la salud a través de la actividad formativa de la enseñanza de la matemáticas en el CGB

\begin{tabular}{|c|c|c|c|c|}
\hline Acción & Finalidad & Logros esperados & Actividades & Tiempo \\
\hline \multirow[t]{2}{*}{$\begin{array}{c}\text { Transformar la } \\
\text { actividad } \\
\text { formativa del } \\
\text { CGB }\end{array}$} & $\begin{array}{l}\text { Incentivar a los } \\
\text { docentes a los } \\
\text { cambios requeridos } \\
\text { para facilitar la } \\
\text { práctica pedagógica } \\
\text { en la easentanza de } \\
\text { la matemática. }\end{array}$ & $\begin{array}{c}\text { Comprensión de los } \\
\text { procesos. } \\
\text { Aceptación de cambios. } \\
\text { Dominio de ambientes de } \\
\text { aprendizajes. } \\
\text { Desarrollo de proyectos de } \\
\text { aprendizaies. }\end{array}$ & $\begin{array}{l}\text {-Elaboración de planes } \\
\text { •Compartir criterios. } \\
\text {-Elaborar descripciones. } \\
\text {-Reunir juicios. } \\
\text {-Discutir los hallazgos. }\end{array}$ & 1 mes \\
\hline & & $\begin{array}{c}\text { Valcración de la } \\
\text { matemática y de la ealud. }\end{array}$ & & \\
\hline $\begin{array}{l}\text { Reorientación } \\
\text { de la práctica } \\
\text { pedagógica }\end{array}$ & $\begin{array}{c}\text { Desarrollar } \\
\text { actividades que } \\
\text { faciliten la } \\
\text { ensetianza y el } \\
\text { aprendizaje de la } \\
\text { matemática dentro } \\
\text { de una práctica que } \\
\text { promueva los } \\
\text { estilos de vida } \\
\text { mejorando la salod. }\end{array}$ & $\begin{array}{l}\text { Asuman las necesidades } \\
\text { del aprendizaje en: } \\
\text {-Hábitos de higiene. } \\
\text {-Ámbito personal y grupal. } \\
\text { - Mejores estilos de vido. } \\
\text {-Hábitos alimenticios. } \\
\text {-Valoración de la salud. } \\
\text {-Uso adecuado de los } \\
\text { servicios sanitarios. } \\
\text {-Utilización de los factores } \\
\text { protectores de la salud. } \\
\text {-Medidas preventivas. }\end{array}$ & $\begin{array}{c}\text {-Planificación } \\
\text {-Evaluación de los procesos } \\
\text { pedagógicos. } \\
\text {-Discusión de maroos adecuodos } \\
\text { para producir cambios. } \\
\text {-Elaboración de actividades que } \\
\text { respondan a los problemas } \\
\text { detectados } \\
\text {-Implementación de uso de } \\
\text { diferentes ambientes de } \\
\text { aprendizajea. }\end{array}$ & $\begin{array}{c}2 \\
\text { meses }\end{array}$ \\
\hline $\begin{array}{l}\text { Trabajo } \\
\text { colabcrativo } \\
\text { para la } \\
\text { promoción de } \\
\text { factorea } \\
\text { protectores de } \\
\text { la salud }\end{array}$ & $\begin{array}{l}\text { Integrar a todos los } \\
\text { entes involucrados } \\
\text { en la actividad } \\
\text { académica y la } \\
\text { acción comunitaria. }\end{array}$ & $\begin{array}{l}\text { Participación en laz } \\
\text { actividades de salud. } \\
\text { Conocimientos de los } \\
\text { programas del área de } \\
\text { salod. } \\
\text { Formación sobre loe } \\
\text { aiveles y modelos para } \\
\text { enseṫar el irea de } \\
\text { matemática y salud. } \\
\text { Dominio de los factores } \\
\text { protectores de la salud. }\end{array}$ & $\begin{array}{c}\text { •Enfoquea del trabajo } \\
\text { cooperativo. } \\
\text { •Discusićn de programas } \\
\text { educativos en el área de salud y } \\
\text { matemática. } \\
\text {-Discusión sobre los efectos } \\
\text { esperedos en la implementación } \\
\text { de los diferentes eepacios. } \\
\text {-Hábitos de condiciones de vida. } \\
\text {-Factores protectares y de } \\
\text { riesgos. } \\
\text {-Vinculación de redes. }\end{array}$ & 1 mes \\
\hline $\begin{array}{l}\text { Utilización de } \\
\text { diferentes } \\
\text { espacios }\end{array}$ & $\begin{array}{c}\text { Brinder } \\
\text { acompañamiento a] } \\
\text { docente para el } \\
\text { desarrollo de una } \\
\text { práctica matemática } \\
\text { en un ambiente } \\
\text { dinämico y } \\
\text { didactico al aire } \\
\text { libre. }\end{array}$ & $\begin{array}{c}\text { Uso de ambiente. } \\
\text { Estrategias innovadoras } \\
\text { Elaboracionea de matrices. } \\
\text { Conformación de espacios } \\
\text { abiertos (parque, canchia } \\
\text { deportiva). } \\
\text { Utilización de la } \\
\text { tecnologio. } \\
\text { Jornadas comunitarias. } \\
\text { Integración de padres, } \\
\text { familiares y sociedad. } \\
\text { Vinculación de las } \\
\text { Asignaturas. } \\
\text { Procesos Practicos. } \\
\text { Jornadas Preveativas. } \\
\text { Deaarrolio de actividades } \\
\text { prácticas, deportivas, } \\
\text { salidas pedagogicas y } \\
\text { recreativas. } \\
\text { Procesos de Socializaciön. } \\
\text { Evaluación de las } \\
\text { Actividades. }\end{array}$ & $\begin{array}{l}\text {-Implementación del uso de } \\
\text { metodologia de ambiente } \\
\text { natural - taller. } \\
\text {-Gujas de trabajo. } \\
\text {-Uso de eztrategia que permitan } \\
\text { trabajar una temática } \\
\text {-Elaboraciones de matrices para } \\
\text { determinar logros. } \\
\text {-Análiaia de información para } \\
\text { medir la coherencia entre la } \\
\text { planificación, actividades e } \\
\text { implantación de la propuesta }\end{array}$ & $\begin{array}{c}2 \\
\text { meses }\end{array}$ \\
\hline
\end{tabular}




\section{6}

\section{Conclusiones}

De acuerdo a los resultados obtenidos en la información recopilada, se elaboran las siguientes conclusiones:

Los procesos de intervención del CGB para el desarrollo delárea de matemática, no se enfoca de acuerdo a las características individuales, debido a que no se considera los entornos familiares, ambientales y comunitarios. Por otra parte, la labor del docente en la estructuración de la planificación se mantiene de una manera general y solamente en las asignaturas que enfocan en los contenidos en el área científica [21-22].

Por consiguiente, el proceso de enseñanza desarrollado no promueve factores protectores para la salud, debido a que no se busca la construcción que fomente los niveles de intervención y modelos, donde se aborden no sólo la consecuencia de las enfermedades, sino también la interrelación causal de los problemas que ocasionan la no utilización de los procesos preventivos de la salud en todos los ámbitos, esto debido a que los estudiantes, siempre visualizan la matemática como una ciencia que se mantiene encerrada en el aula, de esta forma se puede observar que no existe relación alguna entre la fomentación deportiva y las ciencias matemáticas [11]. De igual manera, el proceso de formación de los estudiantes donde solamente se den temas de cálculo procedimental, a manera informativa no promueve la reflexión y el análisis crítico de la información, lo que imposibilita la concientización, el desarrollo de nuevos conocimientos y habilidades que contribuya adoptar, mantener estilos de vida saludables, fortalecer el autoestima, y su potencial para insertarse en la comunidad a través de técnicas participativas que puedan trascender las aulas de clases para ir a compartir procesos de transformación con la familia y la comunidad [23-26].
Las estrategias de facilitación y participación que concibe la práctica pedagógica de la institución objeto de estudio, no se desarrolla asumiendo procesos individuales, sociales y comunitarios que permitan que los estudiantes desarrollen procesos integrales, cooperativos y armónicamente en su beneficio y de su grupo comunitario [25]. A tal efecto, el CGB como ente formador debe asumir diferentes espacios para desarrollar la integración, debido a que es un deber de todos y son corresponsables de esta situación. Por consiguiente, el trabajo integrado donde se incentive al descubrimiento, investigación y se reconozca que en la comunidad existe ambientes de aprendizajes que fortalecerán la planificación y actividades para contribuir en el fortalecimiento de la gestión, favoreciendo la estrategia promocional de calidad de vida y salud desde los ambientes de aprendizajes académicos [27].

Dentro de este marco de referencia, se debe impulsar el desarrollo de intervenciones que generen la participación activa del estudiantado a la comunidad, articulando diferentes áreas de atención y funcionando de manera integrada, para buscar acciones concertadas para mejorar los estilos de vida. Por consiguiente, se debe partir de un proceso reflexivo, colectivo que permita interpretar la realidad y construir procesos de intervención preventiva, donde se profesionalicen a los estudiantes, pero promoviendo los factores protectores de la salud en actividades con las ciencias matemáticas dentro y fuera de la institución [28].

\section{Reflexiones}

El proceso de intervención a través de la propuesta, involucrael aprendizaje cooperativo, los trabajos en grupo, el acompañamiento del docente permite transformar las situaciones detectadas en el área de salud en la institución objeto de estudio. Por tanto, el desarrollo de actividades dentro de diferentes ambientes 
de aprendizajes y la utilización de estrategias innovadoras e integradoras le dará un enfoque integral a la promoción de factores protectores de la salud con el uso de las ciencias matemáticas. A tal efecto, conocer las ventajas que brindan potencializar tanto en el docente, estudiante, padres, familiares, entes institucionales y la comunidad hacia procesos de discusión como método factible para promover el hilo conductor de una enseñanza significativa que le permite al estudiante acercase al saber de las ciencias numéricas por medio del buen desempeño físico que realice el estudiante dentro y fuera del aula [29].

Por otra parte, la exploración de alternativas que favorezcan la formación en grupos de trabajo permite la adquisición de conocimientos impulsados por el descubrimiento, donde cada integrante es un representante de la producción científica que genera las diferentes actividades donde todos deben asumir la responsabilidad de aprender [30]. De igual manera, enmarcar temáticas del área de salud pero que a la vez se otorgue la flexibilidad de tomar otros contenidos que acreciente la enseñanza de la matemática, como un tema principal concebido dentro del aprendizaje dinámico y versátil del estudiante, permitiendo que la relación docente, estudiante, este fomentada dentro del dialogo en concordancia con las experiencias del contexto como conocimiento colectivo para promover estilos de vida que suscita de los ambientes educativos. [31] [32]

Por ello, es elemental que el docente reflexione sobre el enfoque que debe dársele a e proceso de enseñanza y aprendizaje, donde se debe asumir situaciones de la vida real y cotidiana de los estudiantes, si se desea lograr que experimenten y refuercen valores y actitudes de acuerdo a sus propias experiencias, donde una de las ventajas es potenciar en los estudiantes actitudes positivas hacia la prevención de la salud, despertando el interés, por el nuevo conocimiento y propiciar la motivación en la búsqueda de respuestas que beneficie su salud y la de la comunidad [33] y [34].

\section{Recomendaciones}

Para el éxito de la propuesta dentro de un enfoque participativo y motivador, donde se dé un proceso de socialización con las experiencias realizadas se recomienda [35]:

Mantener la integración con todos los entes involucrados para comprender y mejorar la responsabilidad que se tiene en la construcción de estilos de vida saludables.

Que los docentes, asuman los cambios de los procesos tradicionales del aprendizaje para impartir una base pedagógica de la visión de la institución educativa moderna, donde se tiene en cuenta que a través de la experimentación se favorece el desarrollo integral del estudiante.

Utilizar una pedagogía hacia la acción que favorezca la motivación de todos los interesados en la promoción de factores protectores de la salud mediante los nuevos estilos de enseñanza de la matemática. Así, el CGB se convierte en creador de ambientes que beneficie las innovaciones, para fortalecer conductas, disposición de espacios, reconstrucciones conceptuales y la eficacia de los programas académicos en el área de salud.

\section{Referencias}

[1] Ley Orgánica de Educación Superior. República del Ecuador. 2010

[2] N. Pacheco-Carrascal, "La motivación y las matemáticas”, ECOMATEMATICO, vol. 7, no. 1, pp. 149-158, ene. 2016

[3] S.J. Velásquez- Luna, J. L. Celis - Gutiérrez, y C.A. Hernández Suárez, "Evaluación contextualizada como estrategia docente 
para potenciar el desarrollo de competencias matemáticas en Pruebas Saber", ECOMATEMATICO, vol. 8, pp. 33-37, ene. 2017

[4] J.T. Rojas Gómez, "El pensamiento Abstracto a partir de la interdisciplinariedad de las Matemáticas", ECOMATEMATICO, vol. 8, pp. 51-53, ene. 2017.

[5] R. Hernández, C. Fernández y P. Baptista, Metodología de la Investigación, México. Mc. Graw - Hill. Interamericana S. A. 2006.

[6] R. Hernández, C. Fernández y P. Baptista, Metodología de la Investigación, México. Mc. Graw - Hill. Interamericana S. A. 2010

[7] P. Hurtado, Metodología de la Investigación, Caracas - Venezuela. SYPAL. 2003

[8]Universidad Pedagógica Experimental Libertador. Metodología de la Investigación. Caracas - Venezuela. 2016

[9] M.F. Cejas Martínez, D.J. Mendoza Velazco. M. Navarro Cejas, J.L. Rogel Villacis y Y.M. Ortega, Freire, "A PerformanceCentred Competency-Based Approach to Quality University Teaching", Integration of Education, vol. 23, No. 3, 350-365, 2019

[10] J. A. Niño-Blanco, C. A. Hernández-Suárez, y M. Y. Bonilla-González, "Práctica pedagógica, dominio afectivo y procesos matemáticos de los docentes de matemáticas en el nivel de educación básica del sector publico", ECOMATEMATICO, vol. 10, no 1, pp. 19-27, ene. 2019

[11] M. Gómez, "Enseñanza de la Educación Física como Agente de Prevención de la Salud del Educando", Trabajo Especial de Grado no publicado. Universidad Pedagógica Experimental Libertador. Táchira - Venezuela. 2007
[12] D. Mendoza Velazco, M. Cejas Martínez, M. Navarro Cejas, V. Vega Falcón and C. Albán Yánez, C. "Moodle research software: Emotional context in ecuatorian higher education", International Journal of Engineering Research and Technology, vol. 12, no. 9, pp. 1491-1500, 2019

[13] D. Mendoza, J. La Madriz, M. López y V. Ramón, "Research Competencies of HigherEducation Teaching Staff Based on Emotional Intelligence", Mediterranean Journal of Social Sciences, vol 9, No. 5, 41, 2018

[14] J. A. Zamora-Araya, J. Ramirez-Jimenez, y F. Delgado-Navarro, "Uso de herramientas tecnológicas y su impacto en el rendimiento en el curso de Cálculo II de la Universidad Nacional”, ECOMATEMATICO, vol. 11, no. 1, ene. 2020

[15] M. Quero Virla, "Confiabilidad y coeficiente Alpha de Cronbach", Telos, vol. 12, no. 2, pp. 248-252, mayo-agosto, 2010

[16] P. Carvajal, Estrategias Metodologías para Desarrollar la Promoción de la Salud en la Escuela y Comunidad. Trabajo Especial de Grado no publicado. Universidad Rafael Urdaneta. Maracaibo - Venezuela. 2007

17] P. Aristimuño, Elementos para Promover la Salud, Madrid - España. Editorial Paidos. 2005.

[18] M. Minoletti, Programas Secuenciales. Maracaibo - Venezuela. Universidad Rafael Urdaneta. 2007

[19] M. Espejo, La Transversalidad de la Educación para la Salud, Caracas - Venezuela. Universidad Pedagógica Experimental Libertador. 2006

[20] M. Ferriani, "La Escuela como una Visión Interventora en el área de Salud Trabajo", 
Especial de Grado no publicado. Universidad Pedagógica Experimental Libertador. Caracas - Venezuela. 2006

[21] M. Martinez, M. Viveros, M. Cejas, and D. Mendoza, "Continuing Education of the Professors at Universidad Tecnológica Equinoccial (UTE)", Mediterranean Journal Of Social Sciences, vol. 10, no. 2, 2019

[22] D. Mendoza, "La gestión supervisora del directivo para el mejoramiento del desempeño docente", INNOVA Research Journal, vol 3, no. $8,17-25.2018$

[23] R. Cordero, Estilos de Vida para la Prevención de la Salud. Valera - Venezuela. Universidad Valle del Momboy. 2006

[24] M. López, "Educación para la Salud. Caracas Venezuela”, Revista Educativa Caracas, 2005

[25] L. Cerqueira, Aspectos para Fortalecer la Promoción de la Salud. México. Editorial Trillas. 2005

[26] R. Nutbeam, "Estilos de Vida Saludables", Mérida-Venezuela, Revista Educere. 2005

[27] S. Larrauri, Aspectos para Fortalecer la Promoción de la Salud. México. Editorial Trillas. 2005

[29] E. Prieto, Estilos de Vida, Madrid - España. Editorial Muralla S.A. 2004

[30] P. Guarigata, Educación para la Salud, Bogotá - Colombia. Editorial Amnistía. 2005

[31] P. Vázquez, Teoría de la Participación, Madrid - España. Editorial Muralla S.A. 2006

[32] J. Dolors, Informe de la Comisión Internacional Sobre la Educación Para el Siglo XXI, Colombia. Editorial Trillas. 2003
[33] P. Lara, Comportamientos de Riegos, Colombia. Editorial Trillas. 2006

[34] Constitución de la República del Ecuador. Quito, Ecuador. 2008

[35] Consejo Nacional de Planificación. Plan Nacional de Desarrollo/Plan Nacional para el Buen Vivir. Quito. Ecuador. 2017-2021 